\title{
Preliminary Studies on the Feasibility of Addition of Vertex View to Conventional Brain SPECT ${ }^{1}$
}

\author{
C.Y. $\mathrm{Ng}^{2,3}$, N.H. Clinthorne ${ }^{2}$, J.A. Fessler ${ }^{4}$, A.O. Hero ${ }^{4}$, Y. Zhang ${ }^{5}$ and W.L. Rogers ${ }^{2,3}$ \\ ${ }^{2}$ Division of Nuclear Medicine, The University of Michigan, MI48109-0552 \\ ${ }^{3}$ Dept. of Biomedical Engineering, The University of Michigan, MI48109-2125 \\ ${ }^{4}$ Dept. of Electrical Engineering, The University of Michigan, MI48109-2122 \\ ${ }^{5}$ Dept. of Radiology, The University of Michigan, MI48109-0326
}

\begin{abstract}
We have investigated the improvement in resolution and sensitivity for brain imaging which would result by the addition of a single stationary vertex view to the tomographic data. This method has the practical advantage of being relatively inexpensive and easy to implement. The uniform Cramer Rao bound is a plot of the minimum achievable standard deviation for estimating the pixel intensity as a function of the bias gradient length. Uniform CR bound analysis indicated an improvement in performance when the vertex detector is added, especially for centrally located pixels for which improvement is seen over the useful depth for brain imaging. Simulation experiments were done with a simple six slice phantom and with the Hoffman brain phantom. Visual inspection of the reconstructed images showed improved resolution and noise characteristics over reconstructed images without the vertex data. Quantitatively, substantial reduction in mean square error was observed for a plane close to the vertex detector. Improvement reduced as distance from the vertex detector is increased. Background activities inside the field of view of the vertex detector but not the tomograph were represented by several blobs of activity on a plane lying outside the reconstruction volume. This activity was estimated by 3D spline fitting jointly with the image reconstruction process. Adding the vertex view to conventional brain SPECT should lead to improved cortical imaging, and to moderate improvement for deep structures.
\end{abstract}

\section{INTRODUCTION}

The predominant noise in SPECT imaging is Poisson which arises from the random nature of radionuclide decay and limited detection sensitivity of collimated imaging systems. In these systems there is always a compromise between sensitivity and spatial resolution. SPECT system sensitivity can be increased by improving the collimator geometric efficiency which decreases spatial resolution. Cone beam collimation used with conventional rotating Anger cameras is able to increase both resolution and sensitivity by reducing the field of view, but it has the drawback of

\footnotetext{
${ }^{1}$ This work is supported by NIH grant CA32846 from the Department of Health and Human Services and in part by a University of Michigan Bioengineering Program Fellowship.

0-7803-3534-1/97 10.00@1997IEEE
}

incomplete sampling which degrades the image quality [1]. Another approach to increase both resolution and sensitivity is to increase the amount of active detector surface that simultaneously views the organ of interest.

Addition of the vertex view data to the conventional tomographic data set is motivated by the desire to increase both the resolution and sensitivity for SPECT brain imaging, and it has the advantage of being relatively inexpensive and easy to implement. Existing systems can be modified to include the vertex detector without major alternations in hardware and image acquisition software. Substantial improvement in sensitivity for a given resolution can be achieved by positioning the vertex collimator in contact with the head. As the vertical dimension of the head is less than its diameter, the brain on average will be closer to the vertex detector. Hence the vertex data set will have higher resolution and reduced self attenuation when compared to the tomographic data set. Also, since fewer brain pixels are to be estimated along a vertex view projection element, these projections will have higher information content. And finally, since the tomographic data set is complete without the vertex view, portions of the vertex view can be disregarded to eliminate focal areas of uptake which are outside the reconstruction volume without introducing artifacts.

However, there are also challenges that are unique to this imaging geometry. First is the need for a 3-dimensional image reconstruction method. Conventional filtered backprojection (FBP) algorithm cannot incorporate the vertex data set as this sampling geometry does not fulfill the sampling requirement of 3D-FBP. We have adopted a penalized weighted least squares (PWLS) objective in conjunction with the iterative coordinate descent (ICD) algorithm as the image reconstruction method [2]. This algorithm converges rapidly to a global minimum of the objective function, readily incorporates a nonnegativity constraint and local roughness penalty, and easily accommodates the non-standard imaging geometry. Second there is a problem introduced by background activity arising from organs outside the brain (such as salivary glands, lung, heart and the thyroid) that are in the field of view of the vertex detector but not the ring tomograph. This background activity must be estimated as a part of the image reconstruction process, and the system model must be modified to incorporate it. 
Evaluation of new tomographic imaging geometries is a long standing problem which is made even more difficult when different image reconstruction algorithms are to be used. Simulation studies are commonly employed, but the image quality is strongly dependent on the type of reconstruction algorithm used, and by the choice of various algorithm parameters which affects bias and variance. There exists a powerful methodology for studying the intrinsic benefits of different data acquisition methods which decouples the data acquisition process from the reconstruction algorithm. This methodology involves the computation of theoretical lower bounds on the mean square error which is specified by the statistics of the raw data alone. Hero et.al. describes a 'uniform' Cramer Rao bound that is applicable to all estimators whose bias gradient length is less than a threshold and makes possible the comparison of different biased estimators [3]. In this paper, we used the uniform CR bound to assess gain in performance for addition of the vertex data set to conventional tomographic data.

\section{EXPERIMENTAL METHODS}

\section{A. Simulation Experiments}

\section{1) Experimental Setup}

Imaging System: The tomographic system is based on SPRINT II [4]. Collimation is provided by a 10 slit ring aperture which assumes 10 angular positions combined with a slice collimator. Geometric slit resolution at the center of the field of view is $13.4 \mathrm{~mm}$. The geometric efficiency is $9 \times 10^{-4}$ for a point source at the center of the field of view. The detector ring is composed of 11 detectors with 22 bars, 6 $\mathrm{mm}$ wide each. The vertex detector is fitted with a parallel hole collimator with holes of diameter $2.05 \mathrm{~mm}$. The length of the collimator is equal to $2 \mathrm{~cm}$ with a point source efficiency of $5.25 \times 10^{-4}$. The intrinsic resolution of the vertex detector is assumed to be $4 \mathrm{~mm}$ FWHM Gaussian with a system resolution of $13.9 \mathrm{~mm}$ at $10 \mathrm{~cm}$ from the face of the vertex collimator. Both systems model a uniform attenuation of $0.15 \mathrm{~cm}^{-1}$.

Object Model: The first object was used in the simulation experiment and uniform bound experiment. The object is cylindrical, consisted of six slices $1.66 \mathrm{~cm}$ thick and $22.2 \mathrm{~cm}$ in diameter represented by a $32 \times 32$ image matrix of $6.9 \mathrm{~mm}$ pixels. The middle of the top slice is located $2.33 \mathrm{~cm}$ from the face of the vertex collimator. This models the top surface of the brain to be $1.5 \mathrm{~cm}$ from the face of the vertex collimator to allow for the skull and CSF. The object is shown in Fig. 1. A set of noisy projection data with and without the vertex data set was obtained from a sequence of 200 repeated trials assuming $1.79 \times 10^{5}$ counts were detected per SPRINT slice. The regularization parameter was varied to introduce different degrees of bias in the reconstructed image. All quantitative results were based on simulation results for the simple phantom. The second object was the digital Hoffman brain phantom. Ten slices of the Hoffman brain phantom, $64 \times 64$ pixels each were reconstructed. The pixel size was $3.45 \mathrm{~mm}$. Each slice of the phantom is $1 \mathrm{~cm}$ thick and the top slice is $1.5 \mathrm{~cm}$ from the surface of the vertex detector.

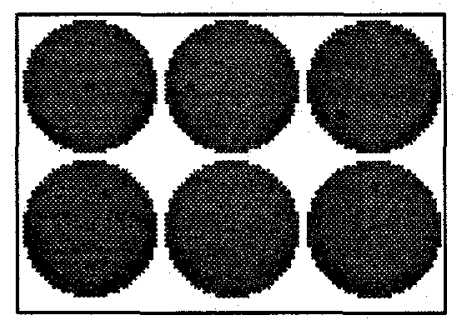

Figure 1: Simple phantom for uniform bound experiments and simulation experiments. Plane 1 (top left) is $2.33 \mathrm{~cm}$ from the vertex detector; plane 6 (lower right) is $11.46 \mathrm{~cm}$ from the vertex detector.

\section{2). Image Reconstruction Algorithm}

The reconstruction algorithm is based on minimizing the following penalized weighted least squares objective function:

$$
\Phi(\underline{\theta})=\frac{1}{2}(\underline{Y}-A \underline{\theta})^{T} K^{-1}(\underline{Y}-A \underline{\theta})+\beta R(\underline{\theta})
$$

In this formulation, $\underline{Y}$ is the projection data, $\underline{\theta}$ is the image and $A$ is the system matrix. The system matrix $A$ for this imaging geometry has the following form:

$$
A=\left[\begin{array}{cccc}
A_{r} & & & \\
& A_{r} & & \\
& & \ddots & \\
& & & A_{r} \\
V_{1} & V_{2} & \cdots & V_{n}
\end{array}\right]
$$

Here, $n$ is the number of slices, $A_{r}$ is the system matrix for the SPRINT ring for each slice of the object. $V_{i}$ is the system matrix for the vertex detector for the $i$ th object slice which is a distance-dependent triangular response function for the vertex collimator convolved with a Gaussian shaped response function of the vertex detector. $K=\operatorname{diag}(Y+0.01 * \max (\underline{Y}))$. Adding $1 \%$ of the maximum of $Y$ stabilizes the solution for projections approaching 0 when we compute the inverse of $K$. Because the image reconstruction problem is ill-posed, we have incorporated a smoothness penalty, $R(\underline{\theta})$, into the least-squares objective which discourages disparities between neighboring pixel values. In this study, we used a twodimensional quadratic penalty of the form:

$$
R(\underline{\theta})=\frac{1}{2} \sum_{j} \sum_{k \in N_{j}} w_{j k} \frac{1}{2}\left(\theta_{j}-\theta_{k}\right)^{2}
$$

where $N_{j}$ is the set of eight neighbors of the $j$ th pixel on the same plane. The weights $w_{j k}$ equals 1 for horizontal and vertical neighbors and $1 / \sqrt{2}$ for diagonal neighbors. A discussion of the convergence properties of PWLS+ICD can be found in [2]. It is a natural requirement for the pixel intensities to be nonnegative, and we included a 
nonnegativity constraint in the algorithm. The ICD algorithm exhibits fast convergence for high spatial frequencies, so if we initialize the reconstruction with a set of 2D FBP images, which gives a reasonable estimate of the low frequencies, then PWLS+ICD converges rapidly.

\section{3). Background}

We found in HMPAO patient studies that activity from salivary gland, thyroid and the chest comprise about $20 \%$ of the total activity detected by the vertex detector but not detected by the ring gantry. To account for this background activity in image reconstruction, we modified the system matrix as follows:

$$
A=\left[\begin{array}{lllll}
A_{r} & & & & \\
& A_{r} & & & \\
& & \ddots & & \\
& & & A_{r} & \\
V_{1} & V_{2} & \cdots & V_{n} & V_{8}
\end{array}\right]
$$

We modeled this background activity to lie on a plane containing several blobs of activity of different intensity and location lying outside the reconstruction volume. This plane is located relatively far away from the vertex detector, hence it will be much blurred by decreased collimator resolution, increased scatter and attenuation. We parameterized this plane with bivariate cubic B-splines on a coarse grid. The vertex weights matrix for the background plane, $V_{g}$, is the transfer function which maps each of the bivariate B-splines onto the detector plane. The task of image reconstruction is then the joint estimation of the object pixel intensities and the coefficients of B-splines for the background plane.

\section{B. The Uniform CR Bound}

In [3], a 'uniform' Cramer-Rao bound was obtained which gives the minimum achievable variance for biased estimators whose bias gradient lengths are less than a pre-specified threshold ' $\delta$ ' where bias gradient length is the norm of the gradient of the bias with respect to $\underline{\theta}$. Instead of constraining the bias gradient vector like the biased form of the classical CR bound, the uniform bound constrains the Cnorm $\|\underline{b}\|_{c}$ of the bias gradient vector. For a non-singular Fisher information matrix, the uniform lower bound and the bias gradient for the parameter $\theta_{1}$ are given by equations (5) and (6) respectively:

$$
\begin{gathered}
B(\underline{\theta}, \delta)=\lambda^{2} \underline{e}_{1}^{T}\left[C^{-1}+\lambda F_{\underline{q}}\right]^{-1} F_{\underline{\theta}}\left[C^{-1}+\lambda F_{\underline{\theta}}\right]^{-1} \underline{e}_{1} \\
\underline{d}_{\min }(\lambda)=-\left[C^{-1}+\lambda F_{\underline{\theta}}\right]^{-1} \underline{e}_{1}
\end{gathered}
$$

$\underline{e}_{l}$ is the unit vector with 1 as the first entry and $\lambda>0$ is given by the unique non-negative solution of the following:

$$
g(\lambda)=\underline{d}_{\min }{ }^{T} \underline{d}_{\min }=\delta^{2}
$$

$\underline{d}_{\min }$ is the optimal bias gradient in the sense that it gives the minimum variance for all bias gradient vectors of the same length. In equations (5) and (6), the matrix $C$ is an arbitrary $n \times n$ symmetric positive definite matrix. $C$ can be chosen to reflect apriori knowledge of the object, where $C=I$ specifies the Euclidean norm. $F_{\theta}$ is the Fisher information matrix. For SPECT the FIM is given by [3]

$$
F_{\underline{\theta}}=A^{T}[\operatorname{diag}(\underline{\mu})]^{-1} A
$$

where $A$ is the system matrix, and $\mu=A \underline{\theta}+\underline{n}$, with $\underline{n}$ being the mean of background counts.

Both $B(\underline{\theta}, \delta)$ and $\underline{d}_{\min }$ are uniquely determined by $\lambda$. To sweep out the bound curve, we only need to compute the value of $B(\underline{\theta}, \delta)$ and $\underline{d}_{\min }$ for several values of $\lambda$. We have implemented the conjugate gradient algorithm to determine the matrix inverse.

\section{Uniform CR Bound Experiment}

Part I: To get a theoretical picture of the gain in performance by the addition of the vertex view to a stationary SPRINT ring, the uniform lower bounds were determined for a center hot pixel of plane 1 from a group of 4 pixels and the single hot center pixel of plane 6 of the phantom in Fig. 1. For this experiment, the imaging parameters were set to be the same as the simulations experiments.

Part II: In this experiment, bounds were calculated to demonstrate the effect of addition of vertex view on central pixels versus peripheral pixels. The two phantoms illustrated in Fig. 2 were used, and both angular and radial sampling were doubled compared to Part $l$, to ensure sufficient sampling for edge-to-center comparison. The dimensions of the phantoms are the same as Part $I$.

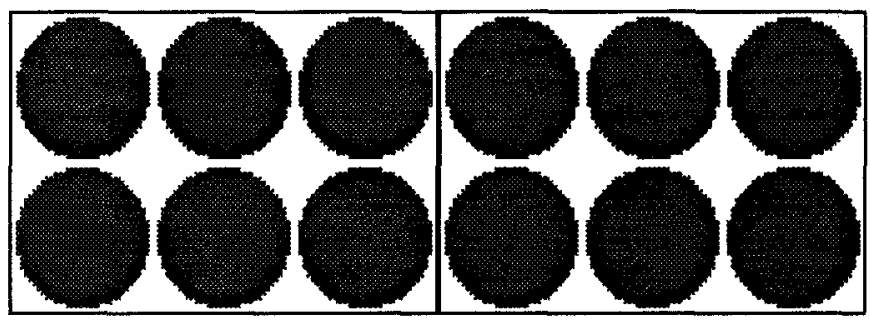

Figure 2: Phantoms for second part of bound experiment Left: Phantom with hot spots at the periphery. Right: Phantom with hot spots near the center

\section{EXPERIMENTAL RESULTS AND DISCUSSION}

\section{A. Simulations \\ 1). Simple Phantom}

Fig. 3 shows the reconstructed image with and without vertex data set. To compare quantitatively the reconstructed images with and without the vertex data set, the bias and variance for a hot pixel at the edge of each of the six planes were determined using the standard method of moments technique. A comparison of the bias and variance trade-off 
for the pixel of interest in the six planes is shown in Fig. 4. The pixels of interest for planes 1, 2 and 3 are the small hot pixel of size 1 at the edge, while for plane 6 , it is one of the pixels from the group of four hot pixels at the edge. The hot edge pixel of planes 1, 2 and 3 are at distances 12.7, 12.1 and 10.5 pixels form the center respectively and they have different bias and variance tradeoff characteristics even for tomograph only data. Pixels that are further away from the center have higher resolution as they are closer to the detector ring and have more favorable bias and variance tradeoff characteristics as demonstrated by the bias and variance curves.

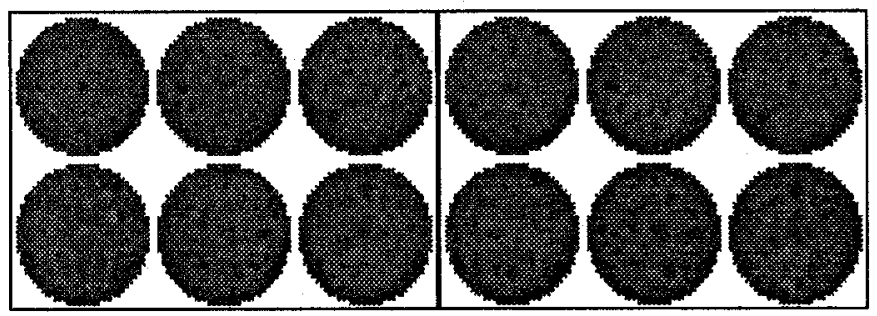

Figure 3: Left: 30 iterations of PWLS reconstruction for ring+vertex data, $\beta$ set to match ratio of geometric resolution. Right: 30 iterations of PWLS reconstruction for ring only data.

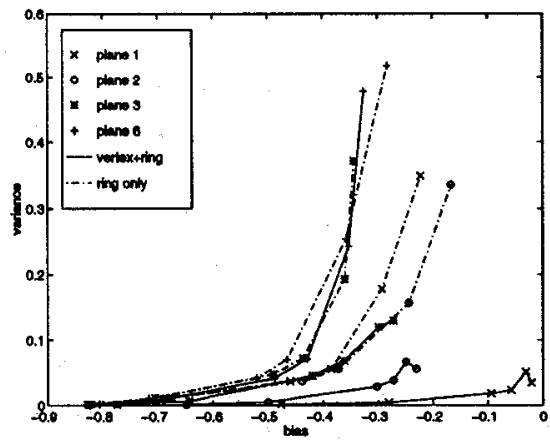

Figure 4: Bias and standard deviation tradeoff characteristics of reconstructed image with and without vertex data.

\section{2). Background}

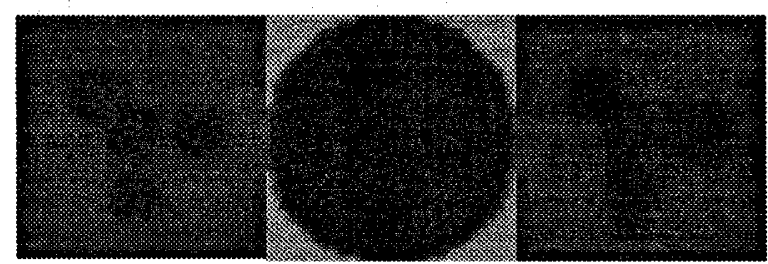

Figure 5: Left: Simulated background plane. Middle: Vertex detector projection data Right: Reconstructed background plane.

Fig. 5 shows the background plane, the projection data of the vertex detector and the reconstructed background plane by spline method. Fig. 6 Left is the reconstructed image with the background plane modeled, Fig. 6 Right is the reconstructed image when the reconstruction algorithm does not model the background plane. The background plane shows up as hot spots on plane 1 of the image, and there are ring artifacts at the edge of the slice indicating mismatch of system model.

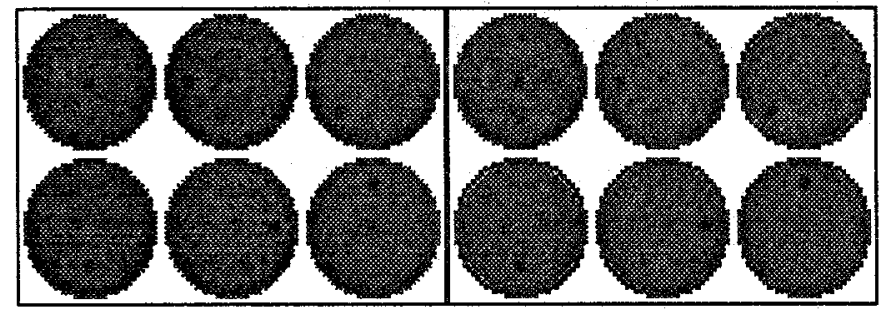

Figure 6: Left: Reconstructed image with background plane modeled. Right: Reconstructed image when reconstruction algorithm does not model background plane.

\section{3) Hoffman Brain Phantom}

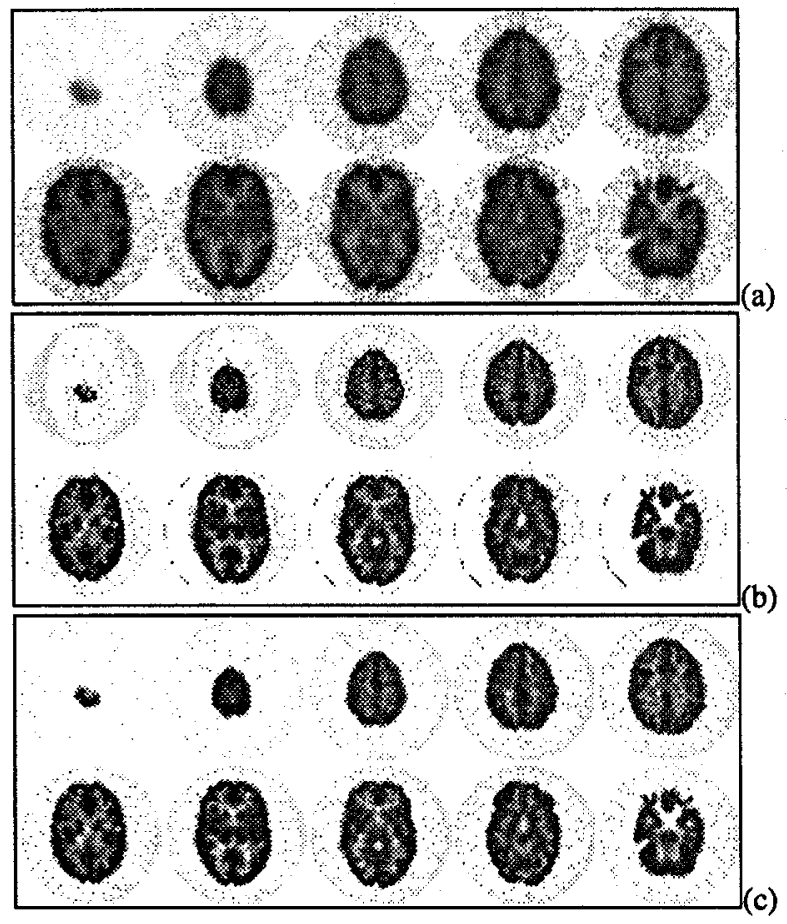

Figure 7(a): Filtered back projection reconstructed image of the digital Hoffman brain phantom. Top row is slices 1 through 5, starting from the left; and bottom row is slices 6 through 10.7(b): 15 iterations of PWLS reconstruction for ring+vertex data, initialized with the FBP image. 7(c): 15 iterations of PWLS for ring only data. $\beta=2 e-8$ for both reconstructions.

Reconstructed images of 10 slices of the digital Hoffman brain phantom, having $64 \times 64$ pixels on each slice, are shown in Fig. 7. For these images an average of 1 million counts are detected per slice of the ring detector. The FBP reconstructed image in Fig. 7(a) was reconstructed with ramp filter only. The images showed streak artifacts typical of FBP algorithm and poor image resolution. Reconstructed image with vertex data showed improved image resolution up to plane 6 which is at a distance of $7 \mathrm{~cm}$ from the surface of the vertex detector. 


\section{B. Uniform CR Bound Experiments}

Part I: Fig. 8 shows the bounds on standard deviation for a center hot pixel of plane 1 and plane $6 . C$ was chosen to be the inverse of the regularization matrix in bound computations. Substantial improvement in performance was obtained when vertex data was added for plane 1. At bias gradient length of 0.6 , the bound was reduced from 3.125 for the ring only case to 0.104 when vertex data is added. The improvement is less prominent for the edge pixels of plane 6 . For bias gradient length greater than 0.55 , there is no difference in performance between the two systems.
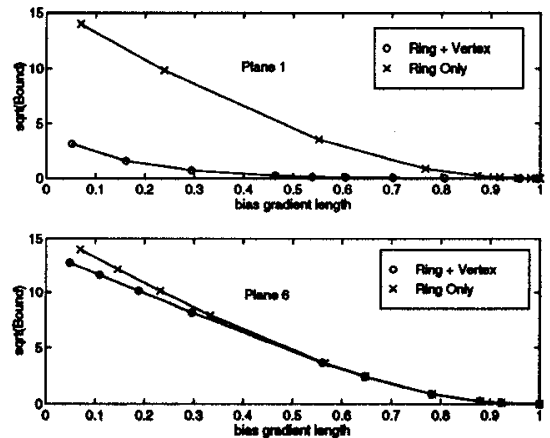

Figure 8: Uniform CR bound for of plane 1 hot center pixel and plane 6 hot edge pixel. $C$ is inverse of regularization matrix.

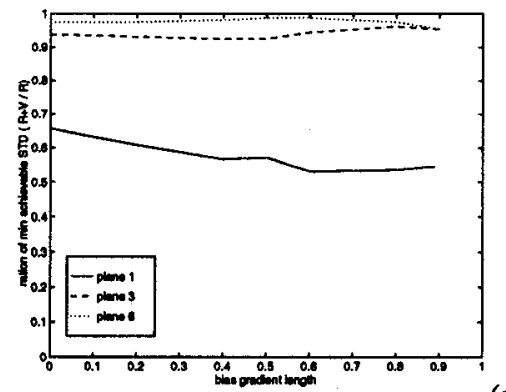

(a)

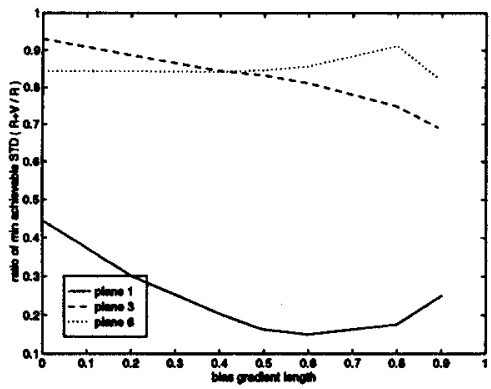

(b)

Figure 9(a): Ratio of sqrt(bound) for a peripheral hot pixel of Ring+vertex to Ring only for planes 1,3 and 6. 9(b) Ratio of sqrt(bound) for a central hot pixel of Ring+vertex to Ring only for planes 1,3 and 6. $C$ is inverse of regularization matrix.

Part II: For Part II of the bound experiments, for the phantom with hot spots at the periphery, addition of the vertex data set resulted in considerable improvement for estimation of regions of interest at plane 1 only. The ratio of the minimum achievable STD for the two cases is less than 0.65 , which is equivalent to a sensitivity increase of 2.8 times. However, the rest of the planes only show marginal improvement when the vertex data set is added (Fig. 9(a)). The ratio of min. achievable STD is very close to 1 for plane 3 and 6. For the phantom with hot spots close to the center, the bound on STD for all the planes is lower for the ring+vertex case when compared to the ring only case. Even at plane 6, the ratio of the minimum achievable STD for the two cases is approximately 0.84 , which is equivalent to a $40 \%$ increase in sensitivity.

\section{CONCLUSIONS}

Using simulation experiments, we have shown that by adding the vertex data set to tomographic data set, the bias and variance trade-off characteristics are superior to standard tomographic data up to distances that are beyond $10 \mathrm{~cm}$ from the vertex detector where the advantage of the vertex view is offset by attenuation effect and decreased vertex resolution. The visual quality of the reconstructed images is also improved for the ring+vertex situation.

Cramer-Rao bound type analysis has been applied very successfully in parameter estimation problems for comparing the optimality of various estimators. In this work, we exploited the application of this type of analysis for performance comparison of medical imaging systems. The introduction of uniform Cramer-Rao bound allows the study of bias-variance and resolution-sensitivity trade-offs for biased image reconstruction algorithms. With this mathematical tool, we are able to examine the intrinsic benefits of these two data acquisition regimes relative to reconstruction accuracy as the data acquisition process is decoupled form the reconstruction algorithm.

It is of interest to note that the vertex view provide the largest benefit to centrally located pixels that are the most poorly measured by the tomograph only. this is probably because peripheral pixels have reduced attenuation and are closer to the aperture for $25 \%$ to $30 \%$ of the projections so that vertex view yields only a marginal increase in information.

\section{BIBLIOGRAPHIC REFERENCES}

[1] R.J. Jaszczak, K.L. Greer, and R.E. Coleman, "SPECT Using a Specially Designed Cone Beam Collimator", Journal of Nuclear Medicine, 29, pp. 1398-1405, 1988.

[2] J.A. Fessler, "Penalized Weighted Least-Squares Image Reconstruction for PET", IEEE Trans. Med. Imag., 13-2, pp. 290-300, June 1994.

[3] A.O. Hero, J.A. Fessler, and M. Usman "Exploring Estimator Bias-Variance Tradeoffs Using the Uniform CR Bound", IEEE Trans. Sig. Proc., vol. SP-44, No. 8, pp. 202641, Aug. 1995.

[4] W.L. Rogers, N.H. Clinthorne, L. Shao, J. Stamos, and K.F. Koral, "SPRINT II, A Second Generation Single Photon Ring Tomograph", IEEE Trans. Med. Imag., 7-4, pp. 291 297, 1988. 